\title{
FDG PET for the Study of Primary Hepatic Malignancies in Children
}

\author{
Rajen J. Mody, MD, ${ }^{1}$ Judith A. Pohlen, $M{ }^{2},{ }^{2}$ Sachit Malde, ${ }^{3}$ Peter J. Strouse, $M D,{ }^{2}$ and Barry L. Shulkin, $\mathrm{MD}^{2 *}$
}

\begin{abstract}
Purpose. This manuscript describes the use of FDG PET in a series of 7 children (11 scans) with primary hepatic malignancies (5 patients with hepatoblastoma, 2 patients with hepatic embryonal rhabdomyosarcoma), together with other imaging (CT and MRI), serum tumor markers, and tumor pathology. Materials and Methods. Seven children with pathologically proven hepatic malignancies underwent 11 FDG PET scans for staging (1 patient) or restaging (6 patients). Tumor uptake of FDG was assessed qualitatively and compared with biochemical and radiological findings. Results. Abnormal uptake was demonstrated in 6 of 7 patients (10 of 11 scans). Three patients subsequently underwent partial hepatic resection, and one underwent brain biopsy, confirming in each that the abnormal uptake of FDG indicated viable tumor. In one patient, intense uptake was due to
\end{abstract}

necrotizing granulomas. In one patient, images were suboptimal due to noncompliance with fasting. Conclusion. Primary hepatic tumors of childhood usually demonstrate increased glycoIytic activity, which allows them to be imaged using PET and the tracer 18F-FDG. The technique is probably most useful for assessing response to therapy, in following AFP (alfa fetoprotein) negative cases and for detecting metastatic disease although a large series of patients will need to be studied to confirm our initial findings. Non neoplastic inflammation may also accumulate FDG and could be confused with malignancy. As these tumors are rare, prospective multicenter studies are necessary to determine the true clinical utility of FDG PET imaging in the management of children with primary hepatic malignancies. Pediatr Blood Cancer (c) 2005 Wiley-Liss, Inc.

Key words: FDG PET; hepatoblastoma; liver; malignancy; pediatric; rhabdomyosarcoma

\section{INTRODUCTION}

FDG PET is an important imaging modality in the management of adult patients with oncologic disorders, and has been approved by the Center for Medicare Service for multiple indications [1]. The role of FDG PET in the evaluation of pediatric malignancies is evolving $[2,3]$. PET holds great promise in assisting the management of childhood tumors, but since these are relatively rare, the precise roles of PET in individual malignancies have not yet been well defined. There is limited literature on the role of FDG PET in primary hepatic malignancies in adults. In children, who have a different spectrum of primary liver tumors as compared to adults, the data are even sparser. In contrast to adults, children with primary hepatic malignancies typically have normal hepatic function and normal hepatic structure in areas not involved with tumor [4-6]. In view of the many differences between adult and pediatric hepatic malignancies, we sought to explore the uptake of FDG in pediatric patients with primary liver tumors and to evaluate the potential clinical utility of FDG PET imaging in children with liver tumors.

\section{MATERIALS AND METHODS}

Seven patients, aged 11 months to 5 years 4 months, underwent FDG PET scanning. There were four boys, three girls. Diagnoses were hepatoblastoma $(n=5)$ and embryonal rhabdomyosarcoma $(\mathrm{n}=2)$. In addition, patients also underwent CT scans, MRI and bone scans as clinically indicated. FDG PET scan was obtained within 2 weeks of concurrent CT, MRI, or bone scan. The FDG PET scan procedure was explained, and written informed consent was obtained from a parent of the pediatric patient. Parents were instructed to maintain the child NPO for $4 \mathrm{hr}$ for scans occurring in the afternoon, or NPO after midnight for scans occurring in the morning. Patients 1-6 received $370 \mathrm{MBq}(10 \mathrm{mCi}) \mathrm{FDG} / 1.7 \mathrm{~m}^{2}$ of body surface

${ }^{1}$ Department of Pediatrics and Communicable Diseases, University of Michigan Health System, B1-G505 University Hospital, 1500 E. Medical Center Drive, Ann Arbor, Michigan 48109-0028

${ }^{2}$ Department of Radiology, University of Michigan Health System, B1G505 University Hospital, 1500 E. Medical Center Drive, Ann Arbor, Michigan 48109-0028

${ }^{3}$ University of Michigan Medical School, B1-G505 University Hospital, 1500 E. Medical Center Drive, Ann Arbor, Michigan 48109-0028

This manuscript describes the results of FDG PET imaging in 7 children (11 scans) with primary hepatic malignancies.

Grant sponsor: General Clinical Research Center; Grant numbers: NCI R01 CA54216, MO1-RR00042.

*Correspondence to: Dr. Barry L. Shulkin, Department of Radiological Sciences, Division of Nuclear Medicine, St. Jude Children's Research Hospital, 332 North Lauderdale, MS \#752, Memphis, TN 38105.

E-mail: barry.shulkin@stjude.org

Received 25 February 2005; Accepted 1 June 2005 


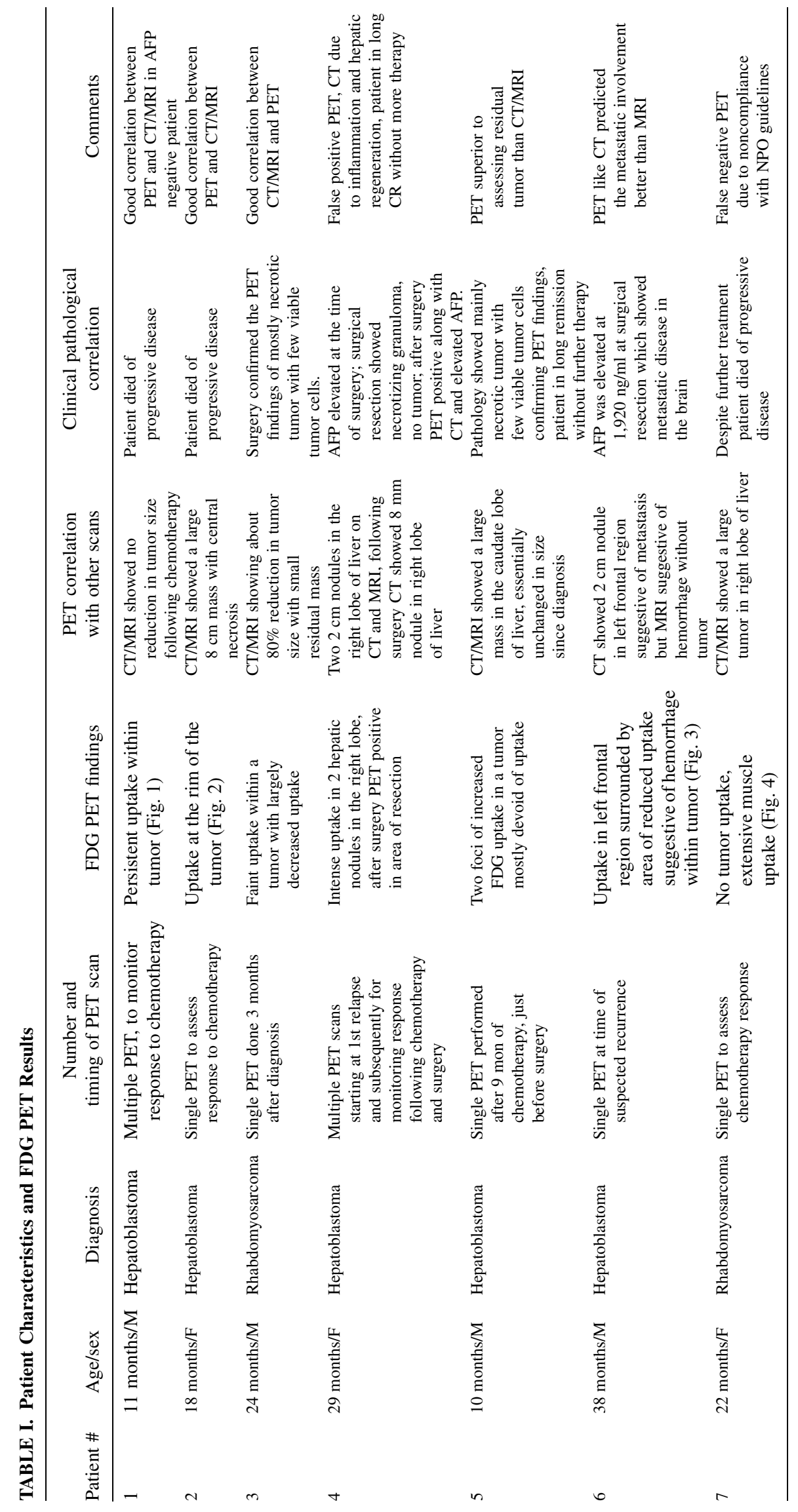


area, and patient 7 received $300 \mathrm{MBq}(8 \mathrm{mCi}) \mathrm{FDG} / 1.7 \mathrm{~m}^{2}$. Patients 1-5 were studied using an ECAT Exact PET scanner (Siemens Medical Systems, Hoffman Estates, Ill). This device contains 24 detector rings and generates 47 transverse planes. Transmission images were obtained using rotating Ge-68 rods. Patients were placed within the PET scanner with the liver centered in the field of view. Positioning was accomplished on the basis of physical findings and in conjunction with findings on CT or MR imaging. In patients 3 and 4, a 10-min transmission scan for attenuation correction was obtained prior to injection, and dynamic images were obtained beginning $5 \mathrm{~min}$ after injection for $10 \mathrm{~min}$ each for $60 \mathrm{~min}$. In patients 1,2 , and 5 , transmission imaging was begun approximately $45 \mathrm{~min}$ after injection, followed by emission imaging. Patient 6 was studied using a Siemens CTI 931 scanner. This device contains 8 rings and generates 15 cross sectional planes. Static images of the head were obtained beginning $45 \mathrm{~min}$ after FDG administration. Patient 7 was studied using a Siemens Biograph PET-CT scanner beginning $45 \mathrm{~min}$ after injection.

Imaging data were reconstructed into transverse crosssectional images by means of filtered back projection and a Hann filter with a cutoff frequency of 0.35 cycles per projection ray, or segmentation with iterative reconstruction. From the transverse images, attenuation-corrected images were constructed in the coronal and sagittal planes. In addition, in most studies, limited "whole-body" views of the chest and abdomen were obtained to screen for metastatic disease. These views consisted of sequential acquisitions covering $30-45 \mathrm{~cm}$ in the z-axis.

Patients 2-7 were sedated prior to imaging in accordance with University of Michigan guidelines. Patients 3 and 4 were sedated prior to injection in order to perform dynamic imaging, and patients 2, 5-7 were sedated after injection of FDG immediately prior to commencement of imaging. Bladder catheters were placed in patients 2-5, 7 to facilitate clearance of FDG from the urinary tract.

\section{RESULTS}

Abnormal uptake of FDG was detected in 6 of 7 patients, in 10 of 11 scans. Table I summarizes the FDG PET results, its correlation with other scans and clinical course of the patient. FDG PET scans in patients 1-3 were highly concordant with CT/MRI findings. In patient 1 FDG PET demonstrated utility in following AFP negative patients (Fig. 1). In patient 2 FDG PET was consistent with persistent disease (Fig. 2) as were CT/ MRI, and AFP ( $>50,000 \mathrm{ng} / \mathrm{ml})$. In patient 3, both FDG PET and CT/MRI showed excellent response to chemotherapy, which was later confirmed by the surgery. In patient 4 FDG PET was falsely positive secondary to inflammatory nodules, which were confirmed by surgery showing granulomatous disease. The CT and MRI were also false positive, both showing nodules corresponding to FDG uptake. Corresponding AFP levels were elevated at $447 \mathrm{ng} / \mathrm{ml}$ (normal $<5 \mathrm{ng} / \mathrm{ml}$ ). Following surgery, AFP was again elevated $(358 \mathrm{ng} / \mathrm{ml})$, and CT/MRI showed abnormal tissue at the margins of previous resection. FDG PET demonstrated uptake in this region. These imaging findings and elevated AFP subsequently resolved without therapy and were thus due to regenerating liver nodules. The patient has remained in complete remission for 2 years without further therapy and last PET, CT, and AFP were normal $(<5 \mathrm{ng} / \mathrm{ml})$.

In patient 5, CT and MRI were essentially unchanged after 9 months of chemotherapy. FDG PET showed only two foci of uptake within the mass. The AFP, quite elevated at diagnosis $(66,000 \mathrm{ng} / \mathrm{ml})$, had fallen to normal values by the time of the FDG PET scan. Surgical resection showed a necrotic tumor with few viable tumor cells. FDG PET scan more accurately displayed the response to chemotherapy as compared to CT/MRI in this case.

In patient 6, FDG PET was obtained on clinical suspicion of relapse and because the CT and MRI had contradictory findings. PET, like CT was suggestive of

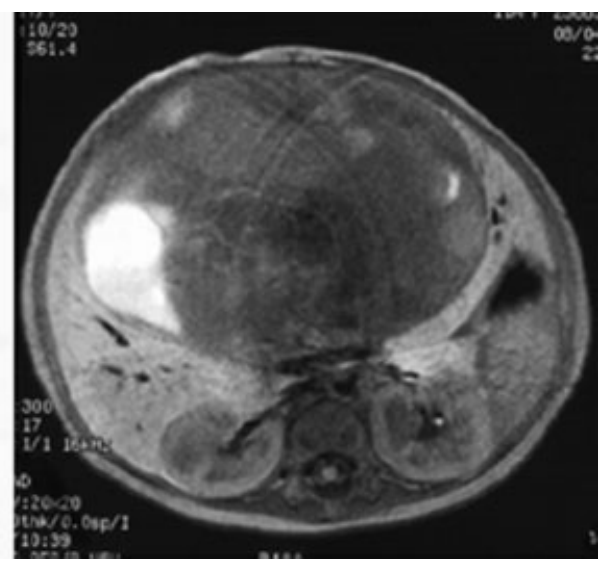

Fig. 1. Transverse image from FDG PET scan (left) shows scattered areas of irregular uptake within the mass in patient 1. Comparison MRI on right shows heterogeneous signal within the mass. Renal activity is shown inferior to the mass. 

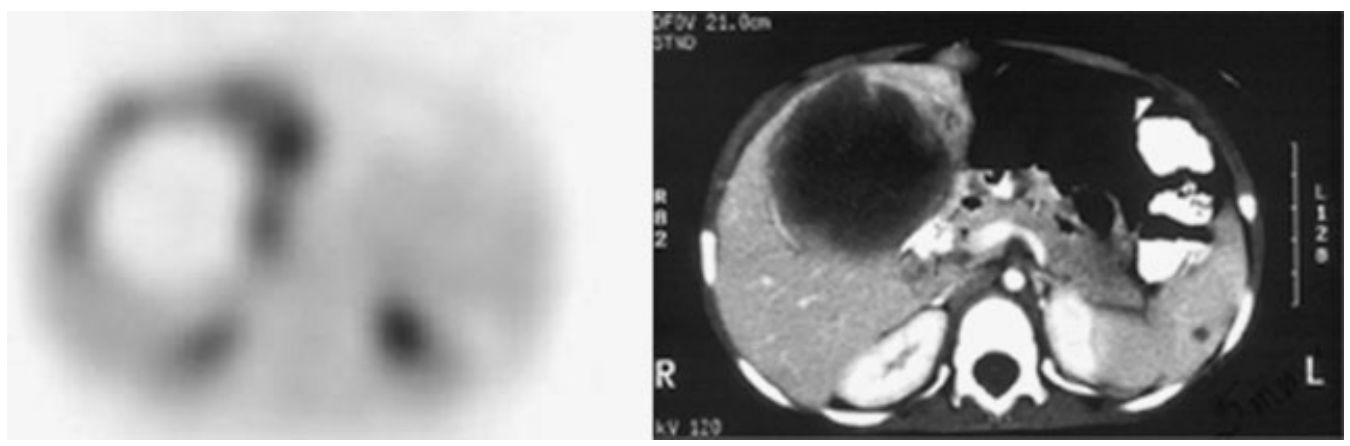

Fig. 2. Transverse image from FDG PET scan (left) shows large area of decreased uptake corresponding with the low density portion of the tumor on CT (right) in patient 2. Along the periphery of the mass are areas of elevated FDG activity representing viable neoplasm.

metastatic focus in the brain, while MRI suggested hemorrhage (Fig. 3). The AFP was elevated $(1,920 \mathrm{ng} / \mathrm{ml})$ and biopsy ultimately concluded that findings were due to metastatic disease (Fig. 3).

Patient 7 had a falsely negative PET scan (Fig. 4) because of noncompliance with NPO guidelines. Images showed extensive muscle uptake, with minimal visceral uptake, particularly in the heart and liver; consequently no tumor was visualized. Review of the CT images obtained in conjunction with the PET scan showed that the stomach was distended with more than $100 \mathrm{cc}$ of liquid. The patient's father acknowledged giving the child apple

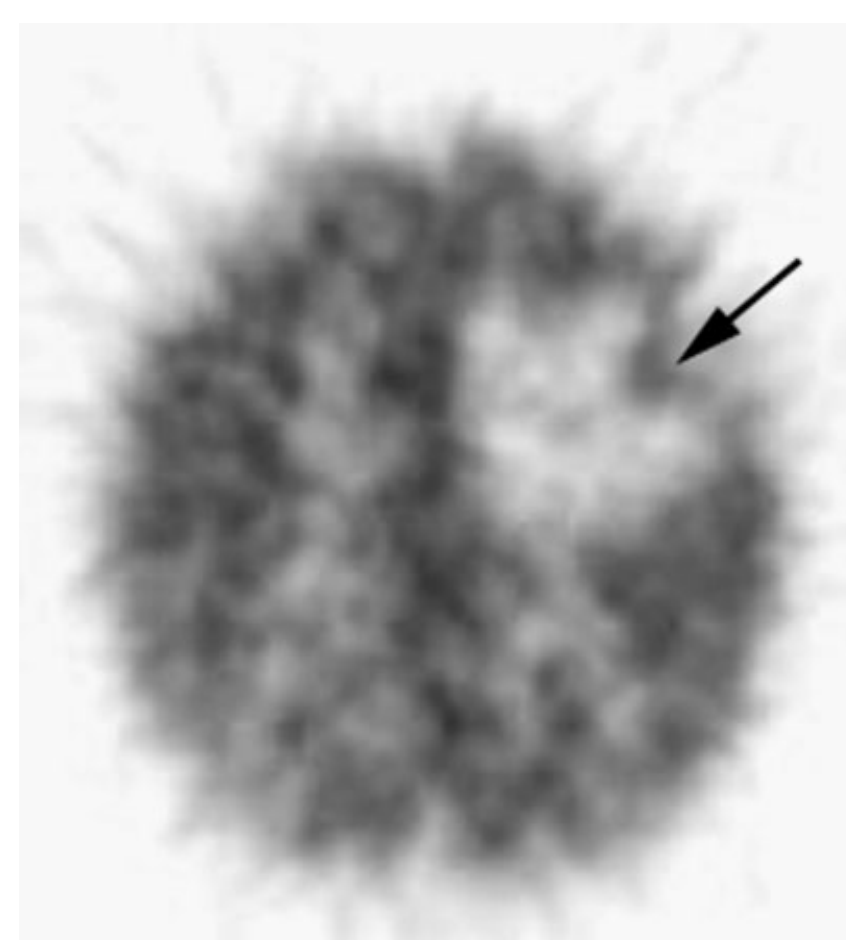

Fig. 3. Transverse image from FDG PET scan of brain shows large area of decreased uptake likely due to localized edema in patient 3 . Within this area is a small region of higher uptake (arrow), but less than that of uninvolved brain, consistent with neoplasm. juice after the injection of FDG. Thus, the most likely explanation for the scan findings is deposition of FDG into muscle from insulin released in response to the carbohydrate load from the apple juice the child drank. A repeat scan was recommended but not performed.

\section{DISCUSSION}

The role of FDG PET scanning in the management of patients with primary hepatic tumors is unclear. Wudel et al. [7] describe a retrospective review of 91 consecutive patients diagnosed with hepatocellular carcinoma. They noted that differentiated hepatocytes normally have a relatively high glucose- 6 phosphatase activity, allowing dephosphorylation of intracellular FDG and its egress

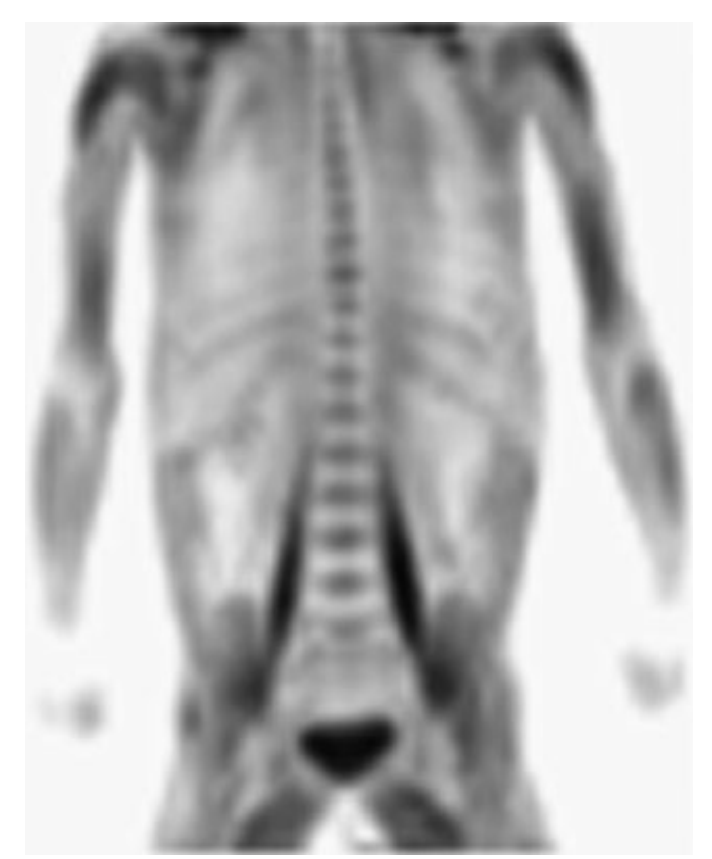

Fig. 4. Anterior projection image from FDG PET scan shows extensive muscle uptake, poor visualization of the liver, and no tumor localization in patient 7 , who was noncompliant with NPO guidelines. 
from the liver. The accumulation of FDG in hepatocellular carcinoma is variable as a result of varying degrees of activity of this enzyme. FDG PET was found to be superior to anatomic imaging in surveillance of patients with metastatic liver disease, post radiofrequency ablation [8].

In our series of 11 scans, 1 was technically suboptimal (due to non-fasting state) and 2 were false positives, with FDG accumulation due to necrotizing granulomas and regenerating liver in the same patient. Seven scans were performed from 9 to 29 days post chemotherapy. Despite the short time intervals between most recent chemotherapy and scanning, FDG avid foci were detected in all these cases. Although we prefer to wait at least 3 weeks following chemotherapy before evaluating tumor response using FDG PET, this was usually not possible due to limited access to the PET device, limited anesthesiology resources, and the challenge of coordinating PET camera availability and anesthesiology availability within a narrow time frame on short notice.

In our series, most of the FDG PET scans showed concordance with CT/MRI results, AFP levels, and postoperative findings. In 3/4 patients (patient \# 3, 5, 6) FDG PET accurately indicated tumor response, while in 1/ 4 patient (patient \# 4) FDG PET was falsely positive. In two patients [1,5], FDG PET detected viable disease when AFP levels were normal. In two patients (patient \# 5, 6) FDG PET predicted the biological nature of the lesions better than CT/MRI, suggesting a role of FDG PET in imaging of these tumors. These clinical scenarios illustrate a very useful role of FDG PET in the management of children with hepatic malignancies.

In summary, our report suggests that hepatic tumors of childhood demonstrate increased glycolytic activity, which allows them to be imaged using PET and the tracer 18F-FDG. FDG PET imaging offers a noninvasive, physiological method to possibly assess extent of disease, response to therapy, and the presence of metastatic disease. However, inflammation and hepatic regeneration may result in false positive findings.

\section{ACKNOWLEDGMENTS}

Supported in part by NCI R01 CA54216 (BS), MO1RR00042 (General Clinical Research Center). The authors appreciate the expertise of Sandra Gaither in preparing this manuscript.

\section{REFERENCES}

1. Maisey MN. Overview of clinical PET. Br J Radiol 2002; S1-S5.

2. Shulkin BL, Mitchell DS, Ungar DR, et al. Malignancy in a pediatric population: 2-[F-18]-fluoro-2-deoxy-D-glucose PET studies. Radiology 1995;194:495-500.

3. Shulkin BL. PET applications in pediatrics. Q J Nucl Med 1997; 41:281-291.

4. Herrera JM, Krebs A, Harris P, et al. Childhood tumors. Surg Clin North Am 2000;80:747-759.

5. Teefey SA, Hildeboldt CC, Dehdashti F, et al. Detection of hepatobiliary malignancy in liver transplant candidates: Prospective comparison of CT, MR imaging, US, and PET. Radiology 2003; 226:553-542.

6. Herzog CE, Andrassay RJ, Eftekhari F. Childhood cancers: Hepatoblastoma. The Oncologist 2000;5:445-453.

7. Wudel LJ, Delbeke D, Morris D, et al. The role of $[18 \mathrm{~F}]$ flurodeoxyglucose positron emission tomography in the evaluation of hepatocellular carcinoma. Am Surg 2003;69:117-124.

8. Anderson GS, Brinkmann F, Soulen MC, et al. FDG positron emission tomography in the surveillance of hepatic tumors treated with radiofrequency ablation. Clin Nuc Med 2003;28:192-197. 